\title{
An Explicit Modular Equation in Two Variables for $\mathbf{Q}(\sqrt{3})$
}

\author{
By Harvey Cohn and Jesse Deutsch*
}

\begin{abstract}
A system of modular equations of norm 2 had been found for the Hilbert modular function field of $\mathbf{Q}(\sqrt{2})$ in an earlier issue of this journal. Here an analogous system is found for $\mathbf{Q}(\sqrt{3})$ but with the help of MACSYMA. There are special difficulties in the fact that two spaces of Hilbert modular functions exist for $\mathbf{Q}(\sqrt{3})$ that can be interchanged by the modular equations. The equations are also a remarkable example of hidden symmetries in the algebraic manifold $\mathbf{V}_{\mathbf{2}}$ which is defined in $\mathbf{C}^{\mathbf{4}}$ by the modular equation.
\end{abstract}

1. Introduction. We extend to the field $\mathbf{Q}(\sqrt{3})$ the modular equation theory that was developed in Cohn [1] for the field $\mathbf{Q}(\sqrt{2})$. The underlying theory of forms and functions was developed by Gundlach [5] so a rapid resume should suffice.

We first define the modular transformation for $\mathbf{Q}(\sqrt{3})$ restricting ourselves to the symmetric case. We now need not one but two spaces:

$$
\begin{aligned}
& \mathbf{H}^{+}: \Im z>0, \Im z^{\prime}>0, \\
& \mathbf{H}^{-}: \Im z>0, \Im z^{\prime}<0 .
\end{aligned}
$$

The ring of integers for $\mathbf{Q}(\sqrt{3})$ is

$$
\mathbf{Z}[\sqrt{3}]=\{\alpha=a+b \sqrt{3} ; a, b \in \mathbf{Z}\} .
$$

Here $\alpha^{\prime}$ denotes the conjugate $(a-b \sqrt{3})$ and the units are generated by

$$
\varepsilon=2+\sqrt{3}
$$

Each of the spaces $\mathbf{H}^{+}$or $\mathbf{H}^{-}$is invariant under the extended Hilbert modular group for $\mathbf{Q}(\sqrt{3})$, namely for

$$
\alpha, \beta, \gamma, \delta \in \mathbf{Z}[\sqrt{3}], \quad \alpha \delta-\beta \gamma=\varepsilon^{u}, \quad u \in \mathbf{Z},
$$

with the corresponding action

$$
\Gamma=\left\{z \rightarrow(\alpha z+\beta) /(\gamma z+\delta), z^{\prime} \rightarrow\left(\alpha^{\prime} z^{\prime}+\beta^{\prime}\right) /\left(\gamma^{\prime} z^{\prime}+\delta^{\prime}\right)\right\}
$$

augmented by the operation of symmetry

$$
\begin{aligned}
& \mathbf{H}^{+}: z \rightarrow z^{\prime}, z^{\prime} \rightarrow z, \\
& \mathbf{H}^{-}: z \rightarrow-z^{\prime}, z^{\prime} \rightarrow-z .
\end{aligned}
$$

Received December 11, 1986; revised April 6, 1987.

1980 Mathematics Subject Classification (1985 Revision). Primary 11F55, 11R11.

Key words and phrases. Hilbert modular functions, modular equation.

*Research supported in part by NSF Grant DMS 8602077 and by Grant No. 666432 from the PSC-CUNY Research Award Program of the City University of New York. 
Next, (symmetric) modular forms are defined for each degree $t \in \mathbf{Z}^{+}$as those functions $G$ such that

$$
G\left(\frac{\alpha z+\beta}{\gamma z+\delta}, \frac{\alpha^{\prime} z^{\prime}+\beta^{\prime}}{\gamma^{\prime} z^{\prime}+\delta^{\prime}}\right) \frac{1}{\left[(\gamma z+\delta)\left(\gamma^{\prime} z^{\prime}+\delta^{\prime}\right)\right]^{t}}=G\left(z, z^{\prime}\right)
$$

equally for both $\mathbf{H}^{+}$and $\mathbf{H}^{-}$also with the appropriate symmetry $(1.4 \mathrm{~b}, \mathrm{c})$. The corresponding linear spaces of modular forms are called $\mathbf{M}_{t}^{+}$and $\mathbf{M}_{t}^{-}$.

The (symmetric) modular forms are generated by Eisenstein series

$$
G_{t}\left(z, z^{\prime}\right)=\sum\left[(\gamma z+\delta)\left(\gamma^{\prime} z^{\prime}+\delta^{\prime}\right)\right]^{-t}, \quad t \geq 1
$$

summed over all pairs $(\gamma, \delta) \neq(0,0)$ restricted so that repetitions by unit multiples are excluded; i.e., if $(\gamma, \delta)$ is present then $(\omega \gamma, \omega \delta)$ is not present in the sum for the unit $\omega \neq 1$. The series converges absolutely for $t>2$, but for $t=1$ or 2 , special summation procedures are required (with which we shall not concern ourselves).

This theory is expounded in detail in Gundlach [5] and we are concerned only with his main result.

THEOREM 1.7. The ring of modular forms for $\mathbf{H}^{+}$under the symmetric group $\Gamma$ is generated by $G_{2}, G_{3}, G_{4}$, while the ring of modular forms for $\mathbf{H}^{-}$is generated by $G_{1}, G_{4}, G_{6}$. In either case, the field of modular functions is generated by $G_{2}^{2} / G_{4}$ and $G_{2}^{3} / G_{6}$.

2. Normalized Generators. We choose generators of the rings

$$
\mathbf{M}^{+}=\bigoplus_{i \geq 1} \mathbf{M}_{t}^{+} \quad \text { and } \quad \mathbf{M}^{-}=\bigoplus_{t \geq 1} \mathbf{M}_{t}^{-}
$$

so as to produce Fourier series with low coefficients missing, just as in the case of modular forms over $\mathbf{Q}(\sqrt{2})$; see Cohn [1], Gundlach [5], and Nagaoka [6]. The Fourier series have terms corresponding to $\alpha \in \mathbf{Z}[\sqrt{3}]$, i.e., $\alpha=a+b \sqrt{3}, a, b \in \mathbf{Z}$. The exponential functions are

$$
\exp \left(2 \pi i \frac{\alpha z-\alpha^{\prime} z^{\prime}}{2 \sqrt{3}}\right)=q^{b} r^{a}
$$

where

$$
q=\exp \left(\pi i\left(z+z^{\prime}\right)\right), \quad r=\exp \left(\pi i\left(z-z^{\prime}\right) / \sqrt{3}\right) .
$$

The spaces $\mathbf{H}^{+}$and $\mathbf{H}^{-}$differ by the restriction on those $\alpha$ which have corresponding terms in the Fourier series expansion:

$$
\begin{array}{ll}
\mathbf{H}^{+}: \alpha=0 \text { or } \alpha>0>\alpha^{\prime} & (\Longleftrightarrow|a| \leq b \sqrt{3}), \\
\mathbf{H}^{-}: \alpha=0 \text { or } \alpha>0, \alpha^{\prime}>0 & (\Longleftrightarrow a \geq|b| \sqrt{3}) .
\end{array}
$$

We can redefine $G\left(z, z^{\prime}\right)$ by a multiplicative constant so we have new Eisenstein series for $\mathbf{H}^{+}$and $\mathbf{H}^{-}$:

$$
G_{t}^{ \pm}=A_{t}^{ \pm}+B_{t}^{ \pm} \sum_{\alpha} q^{b} r^{a} s_{t-1}(a+b \sqrt{3}), \quad t \geq 1,
$$

summed over the restriction (2.3a,b); see Gundlach [5]. Here,

$$
s_{u}(\alpha)=\sum_{\lambda}|N(\lambda)|^{u}(\operatorname{sgn}(N(\lambda)))^{u+1}, \quad u \geq 0,
$$


where the summation is over all ideals $(\lambda) \mid \alpha$ with $\alpha$ (but not $\lambda$ ) restricted by $(2.3 \mathrm{a}, \mathrm{b})$. The constants are found by hand calculation to be the same for $\left(A_{t}^{+}, B_{t}^{+}\right)$ and $\left(A_{t}^{-}, B_{t}^{-}\right)$. They are as follows:

$$
\begin{array}{ccccccc}
t & 1 & 2 & 3 & 4 & 5 & 6 \\
A_{t} & 1 & 1 & 1 & 23 & 1 & 1681 \\
B_{t} & 12 & 24 & 36 & 240 & 12 & 504
\end{array}
$$

Actually, $\left(A_{1}^{+}, B_{1}^{+}\right)$is omitted because $s_{0}(\alpha) \equiv 0$ if $N(\alpha)<0$. $\mathbf{H}^{+}$

We now define normalized generators of $\mathbf{M}_{t}^{+}$and $\mathbf{M}_{t}^{-}$for $t$ even. We define for

$$
\left\{\begin{array}{l}
H_{2}^{+}=G_{2}^{+}=1+24 q(3 / r+4+3 r)+O\left(q^{2}\right), \\
H_{4}^{+}=\left(23\left(G_{2}^{+}\right)^{2}-G_{4}^{+}\right) / 1152=q(1 / r-2+r)+O\left(q^{2}\right), \\
H_{6}^{+}=\left(\left(G_{2}^{+}\right)^{3}-\left(G_{3}^{+}\right)^{2}-432 H_{2}^{+} H_{4}^{+}\right) / 1728=q+O\left(q^{2}\right) .
\end{array}\right.
$$

More detailed expansions are given in Table IA.

Likewise, for $\mathbf{H}^{-}$we define

$$
\left\{\begin{array}{l}
H_{2}^{-}=G_{2}^{-}=\left(G_{1}^{-}\right)^{2}=1+24 r+O\left(r^{2}\right) \\
H_{4}^{-}=\left(23\left(G_{1}^{-}\right)^{4}-G_{4}^{-}\right) / 864=r+O\left(r^{2}\right), \\
H_{6}^{-}=\left(3055\left(G_{1}^{-}\right)^{6}-279\left(G_{1}^{-}\right)^{2}\left(G_{4}^{-}\right)+2 G_{6}^{-}\right) / 311040=r^{2}+O\left(q^{2}\right) .
\end{array}\right.
$$

Again more complete expansions are given in Table IB.

The modular function field is generated by

$$
\begin{array}{ll}
\mathbf{H}^{+}: U=\left(H_{2}^{+}\right)^{2} / H_{4}^{+}, & V=H_{2}^{+} H_{4}^{+} / H_{6}^{+}, \\
\mathbf{H}^{-}: X=\left(H_{2}^{-}\right)^{2} / H_{4}^{-}, & Y=H_{2}^{-} H_{4}^{-} / H_{6}^{-} .
\end{array}
$$

As the expansions suggest, the $q$ - $r$ coefficients of $H_{2}^{ \pm}, H_{4}^{ \pm}, H_{6}^{ \pm}$are all integers (see Deutsch [4]). The proof is similar to the case of $\mathbf{Q}(\sqrt{2})$ (see Cohn [2] and Nagaoka [6]), but requires the Hecke transformation.

3. Modular Equations of Norm 2. We now consider a modular function

$$
w=f / g
$$

where $f$ and $g$ are nonproportional modular forms of degree $t$ (actually, $t=4$ or $6)$. If we transform $z$ by a factor of norm -2 ,

$$
z \rightarrow z(1+\sqrt{3}), \quad z^{\prime} \rightarrow z^{\prime}(1-\sqrt{3})
$$

by analogy with the rational theory and the theory for $\mathbf{Q}(\sqrt{2})$ (see Cohn [1]), there are a total of three conjugates to the transform (3.2a). Namely (3.2a) itself and

$$
\begin{aligned}
& z \rightarrow z /(1+\sqrt{3}), \quad z^{\prime} \rightarrow z^{\prime} /(1-\sqrt{3}), \\
& z \rightarrow(z+1) /(1+\sqrt{3}), \quad z^{\prime} \rightarrow\left(z^{\prime}+1\right) /(1-\sqrt{3}) .
\end{aligned}
$$

In either case, $\mathbf{H}^{+}$and $\mathbf{H}^{-}$are interchanged, so $w$ acquires three conjugates defined over $\mathbf{H}^{-}$or $\mathbf{H}^{+}$as the case may be. We note the transformations in terms of $q$ and $r$ from $(3.2 \mathrm{a}, \mathrm{b}, \mathrm{c})$ by referring to $(2.1)$ :

$$
\begin{aligned}
& q \rightarrow q r^{3}, \quad r \rightarrow q r, \\
& q \rightarrow r^{3 / 2} q^{-1 / 2}, \quad r \rightarrow r^{-1 / 2} q^{1 / 2} \\
& q \rightarrow-r^{3 / 2} q^{-1 / 2}, \quad r \rightarrow-r^{-1 / 2} q^{1 / 2} .
\end{aligned}
$$


Thus, for a form $f=f[q, r]$ we consider three conjugates

$$
\begin{aligned}
f_{\infty} & =f\left[q r^{3}, q r\right], \\
f_{0} & =f\left[r^{3 / 2} q^{-1 / 2}, r^{-1 / 2} q^{1 / 2}\right], \\
f_{1} & =f\left[-r^{3 / 2} q^{-1 / 2},-r^{-1 / 2} q^{1 / 2}\right],
\end{aligned}
$$

and under $(3.2 \mathrm{a}, \mathrm{b}, \mathrm{c})$ the function $w$ in $(3.1)$ has three conjugates

$$
\tilde{w}=\left\{f_{\infty} / g_{\infty}, f_{0} / g_{0}, f_{1} / g_{1}\right\} .
$$

We now define a "norm" and "trace" as modular forms in the alternate space as follows:

$$
\begin{aligned}
N(f) & =f_{\infty} f_{0} f_{1}, \\
T(f, g) & =f_{\infty} g_{0} g_{1}+f_{0} g_{\infty} g_{1}+f_{1} g_{\infty} g_{0} .
\end{aligned}
$$

The trace follows the pattern of the Hecke transformation of index $1+\sqrt{3}$ (see Cohn and Deutsch [3]). So finally, the modular equation for $\tilde{w}$ is

$$
N(g) \tilde{w}^{3}-\tilde{w}^{2} T(f, g)+\tilde{w} T(g, f)-N(f)=0 .
$$

Note the desired norms and traces are of degree 12 for $X$ or $U$ and degree 18 for $V$ or $Y$.

It is not true that under any multiplication $z \rightarrow \mu z, z^{\prime} \rightarrow \mu^{\prime} z^{\prime}$ the corresponding equation for $\tilde{w}$ would have coefficients in $\mathbf{M}^{+}$(or $\mathbf{M}^{-}$). This is true in the present case because symmetry is maintained by $z \rightarrow z(1+\sqrt{3}), z^{\prime} \rightarrow z^{\prime}(1-\sqrt{3})$ since $\frac{1+\sqrt{3}}{1-\sqrt{3}}$ is a unit. The same is true for $z \rightarrow z \sqrt{3}, z^{\prime} \rightarrow-z^{\prime} \sqrt{3}$, but the modular equation of norm three exceeds the time and space limitations of the system. It is likewise infeasible to extend these results to $\mathbf{Q}(\sqrt{5})$ because the minimum (nonunit) norm is four.

4. Derivation of the Modular Equations. We calculated four modular equations for combinations of $f$ and $g$ making up the generating functions in $(2.8 \mathrm{a}, \mathrm{b})$ :

$$
\begin{array}{ll}
\mathbf{M}_{4}^{+}: f=\left(H_{2}^{+}\right)^{2}, g=H_{4}^{+} & (f / g=U), \\
\mathbf{M}_{4}^{-}: f=\left(H_{2}^{-}\right)^{2}, g=H_{4}^{-} & (f / g=X), \\
\mathbf{M}_{6}^{+}: f=H_{2}^{+} H_{4}^{+}, g=H_{6}^{+} & (f / g=V), \\
\mathbf{M}_{6}^{-}: f=H_{2}^{-} H_{4}^{-}, g=H_{6}^{-} & (f / g=Y) .
\end{array}
$$

Series expansions for $H_{2}^{+}, H_{4}^{+}$, and $H_{6}^{+}$were calculated up to $q^{9}$, and for $G_{1}^{-}, H_{2}^{-}$, $H_{4}^{-}, H_{6}^{-}$up to $r^{11}$ using MACSYMA. The functions in the positive spaces had $q-r$ expansions with 156 terms while in the negative spaces there were 78 .

Next, the series expansions for the basis elements

$$
\mathbf{M}_{t}^{ \pm}=\left\langle\left(H_{2}^{ \pm}\right)^{i}\left(H_{4}^{ \pm}\right)^{j}\left(H_{6}^{ \pm}\right)^{k}, 2 i+4 j+6 k=t\right\rangle
$$

were calculated for $t=12$ and 18. It was seen that

$$
\left\{\begin{array}{l}
\operatorname{dim}\left(\mathbf{M}_{12}^{+}\right)=\operatorname{dim}\left(\mathbf{M}_{12}^{-}\right)=7 \\
\operatorname{dim}\left(\mathbf{M}_{18}^{+}\right)=\operatorname{dim}\left(\mathbf{M}_{18}^{-}\right)=12 .
\end{array}\right.
$$


A total of 13 test coefficients (i.e., coefficients of $q^{b} r^{a}$ ) for positive expansions and 14 coefficients for negative expansions were identified. Hence it became an exercise in linear algebra to get the coefficients of $N(g), N(f), T(f, g), T(g, f)$ in terms of the appropriate bases. For example we found these norm forms:

$$
\begin{gathered}
N\left(H_{4}^{+}\right)=\left(H_{6}^{-}\right)^{2}, \\
N\left(H_{4}^{-}\right)=-8\left(H_{6}^{+}\right)^{2}-6 H_{2}^{+} H_{4}^{+} H_{6}^{+}+32\left(H_{4}^{+}\right)^{3}-\left(H_{2}^{+}\right)^{2}\left(H_{4}^{+}\right)^{2}, \\
N\left(H_{6}^{+}\right)=-8\left(H_{6}^{-}\right)^{3}+\frac{3}{2} H_{2}^{-} H_{4}^{-}\left(H_{6}^{-}\right)^{2}-\frac{1}{16}\left(H_{2}^{-}\right)^{3}\left(H_{6}^{-}\right)^{2} \\
-\frac{3}{2}\left(H_{4}^{-}\right)^{3} H_{6}^{-}+\frac{1}{8}\left(H_{2}^{-}\right)^{2}\left(H_{4}^{-}\right)^{2} H_{6}^{-}-\frac{1}{16} H_{2}^{-}\left(H_{4}^{-}\right)^{4}, \\
N\left(H_{6}^{-}\right)=H_{2}^{+}\left(H_{4}^{+}\right)^{4}+4\left(H_{4}^{+}\right)^{3} H_{6}^{+} .
\end{gathered}
$$

With $f_{1}(U, X, Y)$ the modular equation for $\mathbf{M}_{4}^{+}, f_{2}(X, U, V)$ the one for $\mathbf{M}_{4}^{-}$, $f_{3}(V, X, Y)$ the one for $\mathbf{M}_{6}^{+}$and $f_{4}(Y, U, V)$ for $\mathbf{M}_{6}^{-}$, the equations were determined to be as in Table II. The norms $(4.4 \mathrm{a}, \mathrm{b}, \mathrm{c}, \mathrm{d})$ are in evidence in the cubic powers in each function.

5. Symmetries of the Modular Equations. It is the nature of modular functions that a symmetry is built into the modular equations. This occurs because (3.2a) and (3.2b) are inverse operations.

For example, under $(3.2 \mathrm{a}, \mathrm{b}, \mathrm{c}) U \rightarrow \tilde{U}, V \rightarrow \tilde{V}$ we must solve

$$
f_{1}(\tilde{U}, X, Y)=f_{3}(\tilde{V}, X, Y)=0
$$

for $\tilde{U}$ and $\tilde{V}$. This leads to three values of each corresponding to $(3.2 \mathrm{a}, \mathrm{b}, \mathrm{c})$, and this makes nine pairs. As in the case of $\mathbf{Q}(\sqrt{2})$ (see Cohn [1]), only three pairs can be admissible, namely those that satisfy either of these two equations

$$
f_{2}(X, \tilde{U}, \tilde{V})=f_{4}(Y, \tilde{U}, \tilde{V})=0 .
$$

Likewise, if under (3.2a,b,c) $X \rightarrow \tilde{X}, Y \rightarrow \tilde{Y}$, we would be solving (5.2a) with confirmation by $(5.2 \mathrm{~b})$ :

$$
\begin{aligned}
& f_{2}(\tilde{X}, U, V)=f_{4}(\tilde{Y}, U, V)=0, \\
& f_{1}(U, \tilde{X}, \tilde{Y})=f_{3}(V, \tilde{X}, \tilde{Y})=0 .
\end{aligned}
$$

Analogously, in the case of $\mathbf{Q}(\sqrt{2})$ the same phenomenon occurred but $f_{1} \equiv f_{2}$ and $f_{3} \equiv f_{4}$ since only the space $\mathbf{H}^{+}$was used.

The four equations (5.1a,b) define $\mathbf{V}_{2}$, a two-dimensional manifold in the fourspace of $(X, Y, \tilde{U}, \tilde{V})$. (Actually any three of the four must define the same manifold.) If we think of $\mathbf{V}_{2}$ as defined by an ideal in $\mathbf{Q}[X, Y, \tilde{U}, \tilde{V}]$, then $f_{1}, f_{2}, f_{3}, f_{4}$ are elements of the ideal characterized not only by the absence of one variable, but by the fact that one of the remaining three variables is of degree two while the others are of degree three. Thus,

$$
\operatorname{deg}_{Y} f_{1}=\operatorname{deg}_{X} f_{3}=\operatorname{deg}_{V} f_{2}=\operatorname{deg}_{U} f_{4}=2 .
$$

In some sense, this characterization must be unique.

Very likely there is a purely algebraic way of seeing the fact that $f_{1}, f_{2}, f_{3}, f_{4}$ determine the same manifold $\mathbf{V}_{2}$ as any three of them, and indeed the manifold $\mathbf{V}_{2}$ 
is of codimension 2 (not 3 )! Perhaps a more powerful algebraic system will enable us to demonstrate this later on; hopefully also for the system for $\mathbf{Q}(\sqrt{2})$.

For the time being, we submit numerical values which satisfy $f_{j}=0$ for $j=$ $1,2,3,4$. These values can be used to check the equations in the event that any future research requires the reader to correctly copy the four equations!

$$
(X, Y, \tilde{U}, \tilde{V})=(4,5,53.9834902447,-4.0280639935) .
$$

The values $\tilde{U}$ and $\tilde{V}$ are the real roots of the equations

$$
\begin{aligned}
U^{3}-62 U^{2}+82748 U-4443664 & =0 \\
637 V^{3}+8356 V^{2}+56816 V+134912 & =0 .
\end{aligned}
$$

It would be better to find exact rational or radical check values consisting of fixed points, as was possible for the case of $\mathbf{Q}(\sqrt{2})$, but this is much more difficult for $\mathbf{Q}(\sqrt{3})$ and perhaps will be the subject of later computations.

6. The Computation. The computation was made using MACSYMA on three different computer systems, the City College VAX running under UNIX, the University of Arizona Mathematics Department SUN work station also with UNIX, and the University of Arizona's RVAX operating under VMS. Eventually, all calculations done at the University of Arizona were produced on the RVAX as it ran 50 to 100 times faster than the SUN. For example, the SUN was barely able to calculate the product of two conjugates of a modular form $(3.4 a, b, c)$ before running out of space. Indeed, sometimes the machine did run out of space. When it succeeded in such a calculation it would take approximately $1 / 2$ hour of CPU time.

RVAX had no trouble working with the conjugates of a modular form (3.4a,b,c). Generally, one to one and a half megabytes of "list" space was sufficient for the calculations. For example, to obtain the matrix of test coefficients of $\mathbf{M}_{18}^{+}$, it took RVAX 2.72 minutes with 1.05 megabytes of "list" space. For $f=H_{2}^{-} H_{4}^{-}$and $g=H_{6}^{-}$it took a total of 9.4 minutes of CPU time in three different MACSYMA sessions to find the test coefficients of $N(g), N(f), T(f, g)$ and $T(g, f)$, with only one megabyte of "list" space on the RVAX and a modestly increased "fixnum" area. The rest of the calculation to find $f_{4}(Y, U, V)$ took an insignificant amount of time, just 25.5 seconds.

Finally a SPITBOL program was designed to enhance the appearence of the "ground out" modular forms in Tables IA and IB.

The authors wish to thank the Scientific Computing Laboratory of City College (CUNY), the Mathematics Department of the University of Arizona and the Computer Center of the University of Arizona for making MACSYMA available on their respective machines. 


\section{TABLE IA**}

Computer printout of basis forms in $\mathbf{M}^{+}$

$$
\begin{aligned}
& G_{2}^{+}=1+q\{72,(96)\} \\
& +q^{2}\{96,360,288,(672)\} \\
& +q^{3}\{72,288,936,576,1008,(960)\} \\
& +q^{4}\{672,576,1512,1344,2016,1152,(2976)\} \\
& +q^{5}\{288,1008,1344,1872,1440,3456,1728,2736,(2496)\} \\
& +q^{6}\{360,960,2016,1440,4680,2016,4032,3744,5040,2592,(6720)\} \\
& +q^{7}\{96,1008,1152,3456,2016,3600,3648,4464,3168,6912 \text {, } \\
& 4032,5328,(4800)\}
\end{aligned}
$$

$$
\begin{aligned}
G_{3}^{+}= & -q\{108,(288)\} \\
& -q^{2}\{288,1836,4320,(3744)\} \\
& -q^{3}\{108,4320,7884,19008,18360,(23616)\} \\
& -q^{4}\{3744,19008,29484,48960,56160,79488,(59040)\} \\
& -q^{5}\{4320,18360,48960,67608,125280,103680,181440,147960,(180288)\} \\
& -q^{6}\{1836,23616,56160,125280,134028,247968,247104, \\
& 315360,312120,412128,(307008)\} \\
& -q^{7}\{288,18360,79488,103680,247968,259416,394560, \\
& -q^{8}\{19008,59040,181440,247104,394560,471852,734400, \\
& 636480,1003968,885600,1071936,1033344,1313280,(943776)\} \\
& -q^{9}\{7884,79488,147960,315360,401976,734400,637740, \\
1153440,1016280,1387584,1283256,1855008,1340280, & 2056320,1568268,(1913184)\}
\end{aligned}
$$

** Here the power series term $q^{b}\left\{a_{t}, a_{t-1}, \ldots, a_{1},\left(a_{0}\right)\right\}$ denotes $\sum_{j=-t}^{t} a_{t} q^{b} r^{j}$, making use of symmetry $a_{t}=a_{-t}$. 


$$
\begin{aligned}
& G_{4}^{+}=23+q\{2160,(6720)\} \\
& +q^{2}\{6720,140400,319680,(490560)\} \\
& +q^{3}\{2160,319680,1635120,2920320,4747680,(4905600)\} \\
& +q^{4}\{490560,2920320,8987760,14770560,23336640,24917760,(31456320)\} \\
& +q^{5}\{319680,4747680,14770560,33752160,49291200,80559360 \text {, } \\
& 85898880,109412640,(105006720)\} \\
& +q^{6}\{140400,4905600,23336640,49291200,106282800,137229120 \text {, } \\
& 213183360,241997760,308599200,294010560,(358108800)\} \\
& +q^{7}\{6720,4747680,24917760,80559360,137229120,254124000 \text {, } \\
& 340394880,490281120,539542080,735920640,702656640 \text {, } \\
& 840278880,(790608000)\} \\
& +q^{8}\{2920320,31456320,85898880,213183360,340394880 \text {, } \\
& \text { 575218800, 702656640, 1078250880, 1117791360, 1496422080, } \\
& 1525319040,1818996480,1672289280,(2013264960)\} \\
& +q^{9}\{1635120,24917760,109412640,241997760,490281120 \text {, } \\
& \text { 702656640, 1192062960, 1376481600, 1971375840, 2210682240, } \\
& \text { 2797264800, 2807300160, 3593993760, 3276460800, 3829448880, }
\end{aligned}
$$

$$
\begin{aligned}
H_{4}^{+}= & +q\{1,(-2)\} \\
& -q^{2}\{2,4,-10,(8)\} \\
& +q^{3}\{1,10,21,-28,-10,(12)\} \\
& -q^{4}\{8,28,48,-20,-40,56,(-160)\} \\
& +q^{5}\{10,-10,20,170,50,-240,300,-130,(-340)\} \\
& -q^{6}\{4,-12,-40,-50,84,362,112,-210,-40,-186,(-48)\} \\
& -q^{7}\{2,10,56,240,362,-626,-260,442,130,-672,100,-410,(1252)\} \\
& -q^{8}\{28,-160,-300,112,-260,-448,100,-80,4,800,-884224,800,(128)\} \\
& +q^{9}\{21,-56,-130,210,-442,-100,-855,390,770,-588, \\
& -\quad-1066,-146,-210,1640,-131,(1386)\}
\end{aligned}
$$




$$
\begin{aligned}
H_{6}^{+}= & q q^{2}\{1,-12,3,(-8)\} \\
& +q^{3}\{3,96,-42,-96,(330)\} \\
& -q^{4}\{8,42,48,322,-360,756,(-160)\} \\
& +q^{5}\{3,-96,-322,480,1215,-480,-798,96,(4634)\} \\
& -q^{6}\{12,-330,-360,-1215,2652,-165,-3408,1353,360,-651,(9552)\} \\
& +q^{7}\{1,-96,-756,-480,165,-3936,1142,3936,-11199,-10176, \\
& -q^{8}\{42,-160,798,-3408,-1142,7104,-2106,-9488,-16218, \\
& +q^{9}\{96,-756,96,-1353,3936,2106,22176,-12147,-6240, \\
& -48642,17376,-6231,-21408,-42372,-16032,(105147)\}
\end{aligned}
$$

\section{TABLE IB ${ }^{* * *}$}

Computer printout of basis forms in $\mathbf{M}^{-}$

$$
\begin{aligned}
G_{1}^{-}= & +12 r \\
& +r^{2}\{12,(12)\} \\
& +12 r^{3} \\
& +r^{4}\{12,24,(12)\} \\
& +r^{5}\{24,0,(24)\} \\
& +r^{6}\{12,0,0,(12)\} \\
& +r^{7}\{12,0,24,0,(24)\} \\
& +r^{8}\{12,24,24,24,(12)\} \\
& +12 r^{9} \\
& +r^{10}\{24,24,24,0,24,(24)\} \\
& +r^{11}\{24,0,24,0,24,0,(0)\}
\end{aligned}
$$

${ }^{* * *}$ Here the power series term $r^{b}\left\{a_{t}, a_{t-1}, \ldots, a_{1},\left(a_{0}\right)\right\}$ denotes $\sum_{j=-t}^{t} a_{t} r^{a} q^{j}$, making use of symmetry $a_{t}=a_{-t}$. 


$$
\begin{aligned}
& G_{4}^{-}=23+240 r \\
& +r^{2}\{240,(17520)\} \\
& +r^{3}\{60480,(181680)\} \\
& +r^{4}\{17520,527520,(1123440)\} \\
& +r^{5}\{527520,2877120,(3750240)\} \\
& +r^{6}\{181680,3931200,8951040,(13262640)\} \\
& +r^{7}\{240,2877120,12156960,26282880,(28236000)\} \\
& +r^{8}\{1123440,12156960,38508960,54475680,(71902320)\} \\
& +r^{9}\{60480,8951040,44150400,81768960,132935040,(132451440)\} \\
& +r^{10}\{3750240,38508960,93364320,187012800,219041760,(273767520)\} \\
& +r^{11}\{527520,26282880,93364320,224259840,310807200 \text {, } \\
& 443620800,(425813760)\} \\
& \begin{aligned}
G_{6}^{-}= & 1681+504 r \\
& +r^{2}\{504,(532728)\}
\end{aligned} \\
& +r^{3}\{4058208,(29883672)\} \\
& +r^{4}\{532728,187132176,(545530104)\} \\
& +r^{5}\{187132176,2678616864,(4921875504)\} \\
& +r^{6}\{29883672,4159663200,19805530752,(31587041304)\} \\
& +r^{7}\{504,2678616864,34949354832,107049273408,(142367526000)\} \\
& +r^{8}\{545530104,34949354832,197798710032,425676536208 \text {, } \\
& \text { (558622843128)\} } \\
& +r^{9}\{4058208,19805530752,239637182400,791515839744, \\
& 1506788281152,(1764601070904)\} \\
& +r^{10}\{4921875504,197798710032,1044828083376,2745582285600, \\
& 4328019490032,(5202422407728)\} \\
& +r^{11}\{187132176,107049273408,1044828083376,3814466173056, \\
& 7754664733200,11890620957600,(13072624338816)\}
\end{aligned}
$$




$$
\begin{aligned}
H_{4}^{-}= & +r^{2}\{1,(4)\} \\
& -r^{3}\{24,(-21)\} \\
& +r^{4}\{4,-10,(-80)\} \\
& -r^{5}\{10,-120,(-170)\} \\
& +r^{6}\{21,96,-240,(84)\} \\
& +r^{7}\{1,120,-130,-336,(626)\} \\
& -r^{8}\{80,130,40,442,(-64)\} \\
& -r^{9}\{24,240,-144,-672,-240,(855)\} \\
& +r^{10}\{170,-40,410,-480,770,(680)\} \\
& -r^{11}\{10,336,-410,672,1066,-600,(-1200)\} \\
& +r^{2}-r^{3}\{2,(8)\} \\
& +r^{4}\{1,8,(36)\} \\
& +r^{5}\{8,-38,(-40)\} \\
& -r^{6}\{8,-8,-40,(35)\} \\
& -r^{7}\{38,8,172,(-328)\} \\
& +r^{8}\{36,-8,286,-328,(720)\} \\
& -r^{9}\{2,-40,84,-848,-68,(1848)\} \\
& -r^{10}\{40,-286,896,2152,-520,(-1594)\} \\
& +r^{11}\{8,-172,-896,3112,-1448,-766,(1336)\}
\end{aligned}
$$

\section{TABLE II}

Computer printouts of the modular equations

$f_{1}(u, x, y), f_{2}(x, u, v), f_{3}(v, x, y), f_{4}(y, u, v)$

$$
\begin{aligned}
f_{1}(u, x, y)= & \left(-x^{3}-144 x^{2}-5184 x\right) y^{2}+\left(-3456 x^{2}-248832 x\right) y-2985984 x \\
& +\left(\left(2 x^{2}+288 x+10368\right) y^{2}+\left(-207 x^{2}-9504 x\right) y+62208 x\right) u \\
& +\left(\left(-x^{2}+78 x\right) y-432 x\right) u^{2}+x u^{3}
\end{aligned}
$$

$$
\begin{aligned}
f_{2}(x, u, v)= & \left(-u^{3}+864 u^{2}-186624 u\right) v^{2}+\left(3456 u^{2}-1492992 u\right) v-2985984 u \\
& +\left(\left(-87 u^{2}+2592 u\right) v^{2}+\left(-414 u^{2}-20736 u\right) v-124416 u\right) x \\
& +\left(\left(u^{2}-72 u\right) v^{2}+\left(4 u^{2}-432 u\right) v-1728 u\right) x^{2} \\
& +\left((-u+32) v^{2}-6 u v-8 u\right) x^{3}
\end{aligned}
$$




$$
\begin{aligned}
& f_{3}(v, x, y)=(\left.16 x^{2}+1152 x\right) y+27648 x+\left(-20 x y^{2}+\left(20 x^{2}+96 x\right) y+13824 x\right) v \\
&+\left(4 y^{3}+(-12 x+96) y^{2}+\left(8 x^{2}-160 x\right) y+2304 x\right) v^{2} \\
&+\left(y^{3}+(-2 x+24) y^{2}+\left(x^{2}-24 x\right) y+128 x\right) v^{3} \\
& f_{4}(y, u, v)=\left(u^{2}-464 u+13824\right) v^{3}+\left(6 u^{2}-4320 u+55296\right) v^{2} \\
&+\left(8 u^{2}-13824 u\right) v-13824 u \\
&+\left((71 u+1728) v^{3}+(360 u+6912) v^{2}+432 u v\right) y \\
&+\left((-u+72) v^{3}+(-2 u+288) v^{2}\right) y^{2}+\left(v^{3}+4 v^{2}\right) y^{3}
\end{aligned}
$$

Mathematics Department

City College (CUNY)

New York, New York 10031

Mathematics Department

University of Arizona

Tucson, Arizona 85721

1. H. СонN, "An explicit modular equation in two variables and Hilbert's twelfth problem," Math. of Comp., v. 38, 1982, pp. 227-236.

2. H. CонN, Successive Diagonal Projections of Hilbert Modular Functions, New York Number Theory Seminar, Springer Lecture Notes in Math., vol. 1240, 1987, pp. 69-86.

3. H. COHN \& J. DEUTSCH, "Application of symbolic manipulation to Hecke transformations of modular forms in two variables," Math. of Comp., v. 48, 1987, pp. 139-146.

4. J. I. DeutSCH, Identities on Modular Forms in Several Variables Derivable from Hecke Transformations, Dissertation, Brown University, 1986.

5. K. B. GUNDLACH, "Die Bestimmung der Funktionen zu einigen Hilbertschen Modulgruppen," J. Reine Angew. Math., v. 220, 1965, pp. 109-153.

6. S. NAGAOKA, "On Hilbert modular forms III," Proc. Japan Acad., v. 59, 1983, pp. 346-348. 